\title{
Serotonin toxicity: a practical approach to diagnosis and treatment
}

\author{
Geoffrey K Isbister, Nicholas A Buckley and Ian M Whyte
}

W

ith the introduction of numerous serotonergic agents in the past two decades, serotonin toxicity ${ }^{1}$ has become an important and common adverse drug effect, which can be mild, moderate or even life-threatening. Serotonin toxicity can occur from an overdose, drug interaction or adverse drug effect involving serotonergic agents. Selective serotonin reuptake inhibitors (SSRIs) are one of the commonest groups of drugs taken in overdose, ${ }^{2}$ and serotonin toxicity occurs in $15 \%$ of SSRI overdoses. ${ }^{3}$ A growing list of medications has been associated with serotonergic toxicity; the drugs that have been clearly associated are given in Box 1. The potential for the development of serotonin toxicity is particularly important in patients being prescribed psychotropic medications, with an increasing proportion of the population taking the newer antidepressants. ${ }^{4}$

Serotonin toxicity results from an excess of serotonin (5-hydroxytryptamine [5-HT]) in the central nervous system (CNS), which can be due to several different pharmacological mechanisms. These include inhibition of the metabolism of serotonin (monoamine oxidase inhibitors), prevention of the reuptake of serotonin in nerve terminals (serotonin reuptake inhibitors), and increased serotonin precursors (tryptophan) or serotonin release (serotonin-releasing agents) (Box 2). The resulting excess CNS serotonin acts on serotonin receptors and produces the clinical effects. The exact role of the various serotonin receptors is not completely clear, but there is good evidence that the severe life-threatening clinical effects, such as rigidity and hyperthermia, are mediated by the $5-\mathrm{HT}_{2 \mathrm{~A}}$ receptors. ${ }^{1,5}$

Serotonin toxicity is sometimes called the serotonin syndrome, which is often used to refer to clinical effects "as defined by the Sternbach criteria". 6 However, these criteria were stated to be provisional, are non-specific and have never been validated (see Diagnosis of serotonin toxicity). The clinical features reported in the original description of the serotonin syndrome by Sternbach have often been taken as diagnostic criteria, which has led to some reported associations with medications that clearly do not cause serotonin excess. The atypical antipsychotic drugs, such as olanzapine, are a striking example. These have been reported as causing the serotonin syndrome, ${ }^{7}$ despite having antiserotonergic actions. We contend that the diagnostic criteria developed more recently by our group - the Hunter Serotonin Toxicity Criteria ${ }^{2}$ — are much more specific for serotonin toxicity.

\section{Clinical features of serotonin toxicity}

Serotonin toxicity is characterised by the presence of a triad of clinical features: neuromuscular excitation, autonomic stimulation, and changes in mental state (Box 3). There are a number of specific neurological signs that are not seen in many other conditions that should direct the clinician towards a diagnosis of serotonin toxicity. The most important is generalised hyperreflexia. Sustained clonus is usually found at the ankle; ocular clonus (or non-directional nystagmus) is also very common. Generalised spontaneous clonus may occur in moderate-to-severe cases, and is seen rarely in any condition other than serotonin toxicity. ${ }^{2}$ The

\section{ABSTRACT}

- Excess serotonin in the central nervous system leads to a condition commonly referred to as the serotonin syndrome, but better described as a spectrum of toxicity - serotonin toxicity.

- Serotonin toxicity is characterised by neuromuscular excitation (clonus, hyperreflexia, myoclonus, rigidity), autonomic stimulation (hyperthermia, tachycardia, diaphoresis, tremor, flushing) and changed mental state (anxiety, agitation, confusion).

- Serotonin toxicity can be: mild (serotonergic features that may or may not concern the patient); moderate (toxicity which causes significant distress and deserves treatment, but is not life-threatening); or severe (a medical emergency characterised by rapid onset of severe hyperthermia, muscle rigidity and multiple organ failure).

- Diagnosis of serotonin toxicity is often made on the basis of the presence of at least three of Sternbach's 10 clinical features. However, these features have very low specificity. The Hunter Serotonin Toxicity Criteria use a smaller, more specific set of clinical features for diagnosis, including clonus, which has been found to be more specific to serotonin toxicity.

- There are several drug mechanisms that cause excess serotonin, but severe serotonin toxicity only occurs with combinations of drugs acting at different sites, most commonly including a monoamine oxidase inhibitor and a serotonin reuptake inhibitor. Less severe toxicity occurs with other combinations, overdoses and even single-drug therapy in susceptible individuals.

- Treatment should focus on cessation of the serotonergic medication and supportive care. Some antiserotonergic agents have been used in clinical practice, but the preferred agent, dose and indications are not well defined.

MJA 2007; 187: 361-365

lower limbs usually have a much greater degree of hyperreflexia and clonus than the upper limbs ${ }^{8}$ (although a mechanism for this consistent observation is not known).

Some mental state and autonomic features are also nearly always present, but have lower diagnostic utility, as they are indistinguishable from those observed with other causes of an agitated delirium. However, the presence of these features is often associated with moderate-to-severe serotonin toxicity.

\section{Assessment of serotonin toxicity: diagnosis and severity}

The assessment of serotonin toxicity requires determining, first, whether the clinical features are consistent with serotonin toxicity and, second, the severity of the toxicity. In patients with suspected serotonin toxicity, the clinical assessment should include observation for tremor, myoclonic jerks, diaphoresis, ocular clonus and 


1 Drugs that have been associated with serotonin
toxicity
Serotonin reuptake inhibitors
- Selective serotonin reuptake inhibitors: fluoxetine, fluvoxamine,
paroxetine, citalopram, sertraline, escitalopram
- Other antidepressants: venlafaxine, clomipramine, imipramine
- Opioid analgesics: pethidine, tramadol, fentanyl,
dextromethorphan
- St John's wort
Monoamine oxidase inhibitors
- Irreversible monoamine oxidase A inhibitors: phenelzine,
tranylcypromine
- Reversible monoamine oxidase A inhibitors: moclobemide
- Others: linezolid
Serotonin-releasing agents
- Fenfluramine
- Amphetamines
- Methylenedioxymethamphetamine (MDMA; ecstasy)
Miscellaneous
- Lithium

agitation. Vital signs (heart rate, blood pressure and temperature) will usually be sufficient to diagnose autonomic features. However, most important is a focused neurological examination - mental state (eg, orientation, concentration, short-term memory); upperand lower-limb tone, clonus and reflexes; and pupillary size, reaction and extraocular movements (opsoclonus). In most cases this is sufficient to make a confident diagnosis. ${ }^{2}$

\section{Diagnosis of serotonin toxicity}

A number of diagnostic criteria have been suggested for serotonin toxicity. The first and most commonly used are Sternbach's criteria. ${ }^{6}$ Many of the 10 clinical features suggested as typical of serotonin toxicity by Sternbach are non-specific. These would also be commonly observed in many other conditions such as anticholinergic delirium, and alcohol and drug withdrawal states. ${ }^{2}$ Sternbach's clinical definition was based on case reports and small published case series. Sternbach recognised that the features were non-specific and specified that other possible causes of the features must be excluded. Unfortunately, this caveat on the original description is almost routinely ignored. Thus, while Sternbach's criteria remain useful for early recognition, they cannot by definition be used in differential diagnosis. ${ }^{1}$

As the clinical features of Sternbach's criteria are not specific, they are also not useful for identifying new drugs as a cause of serotonin toxicity. Again, by definition they state that a known serotonergic drug must have been recently added or increased in dose. The folly of ignoring these two components of the syndrome definition is well illustrated by the fact that eight of the 10 "diagnostic" clinical features of the Sternbach serotonin syndrome are common features of the serotonin reuptake inhibitor discontinuation syndrome, and five are in the proposed diagnostic criteria for that syndrome. ${ }^{9}$ In addition, eight of Sternbach's 10 "diagnostic" features can occur with catecholamine excess, and many features are found in other common toxidromes.

We recently developed diagnostic criteria for serotonin toxicity - the Hunter Serotonin Toxicity Criteria (HSTC) — by studying large numbers of patients ingesting serotonergic agents in over-

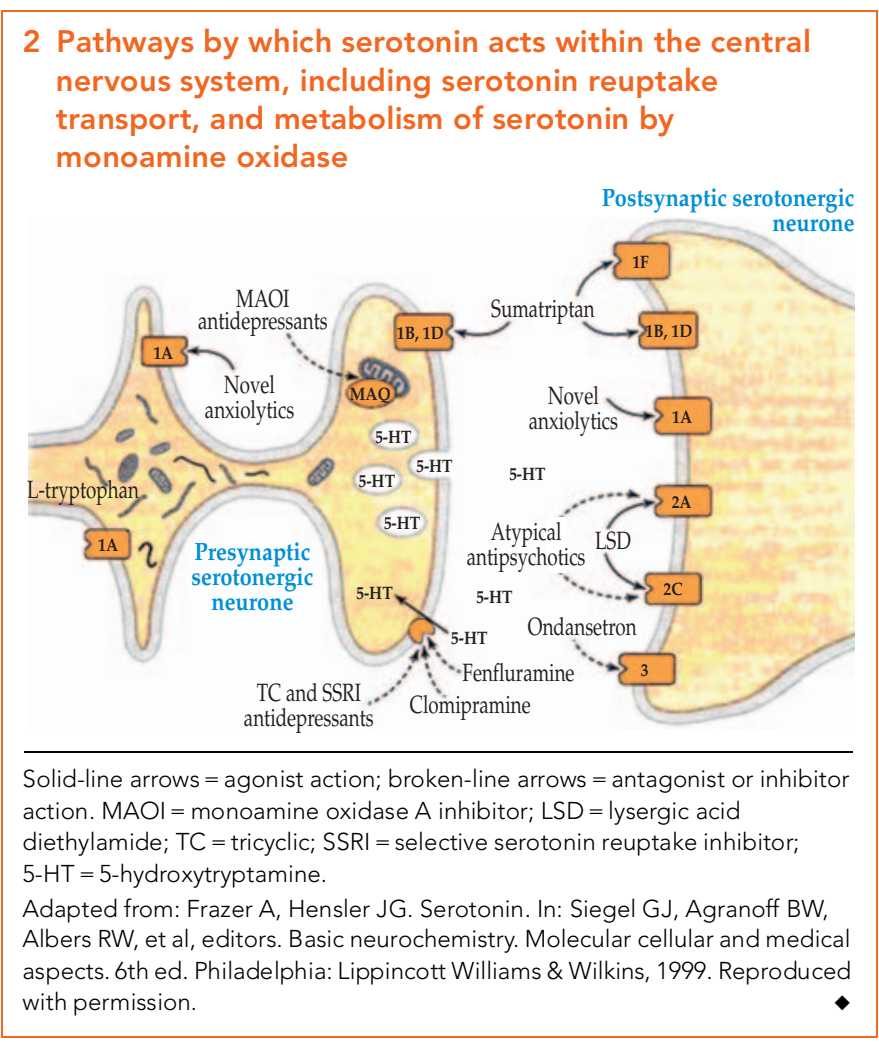

dose. The HSTC can be used to determine whether a patient who has taken an overdose has significant serotonin toxicity (Box 4). ${ }^{2}$ As the HSTC were validated in the setting of a toxicology service where other drug-induced syndromes are frequent, the criteria are much more specific than Sternbach's criteria for features that solely relate to serotonin toxicity. Because they are more specific, the HSTC can be used for adverse drug reactions, but have not been validated for this purpose.

\section{Differential diagnosis}

Although other adverse drug reactions can be initially mistaken for serotonin toxicity, a careful examination for specific neurological features, such as clonus, hyperreflexia and tone, makes it possible to distinguish other conditions. A list of differential diagnoses is given in Box 5. The most commonly confused neurotoxic syndrome is the neuroleptic malignant syndrome. However, neuroleptic malignant syndrome is associated with bradykinesia, lead pipe rigidity, and other extrapyramidal features, in contrast to the hyperkinesia, hyperreflexia and clonus seen with serotonin toxic-

3 Clinical features of serotonin toxicity

Neuromuscular effects Autonomic effects Mental state changes

\begin{tabular}{lll}
\hline - Hyperreflexia & - Hyperthermia: & $\bullet$ Agitation \\
- Clonus & mild,$<38.5^{\circ} \mathrm{C} ;$ & $\bullet$ Hypomania \\
- Myoclonus & severe $\geqslant 38.5^{\circ} \mathrm{C}$ & - Anxiety \\
- Shivering & - Tachycardia & - Confusion \\
- Tremor & - Diaphoresis & \\
- Hypertonia/rigidity & - Flushing & \\
& - Mydriasis
\end{tabular}




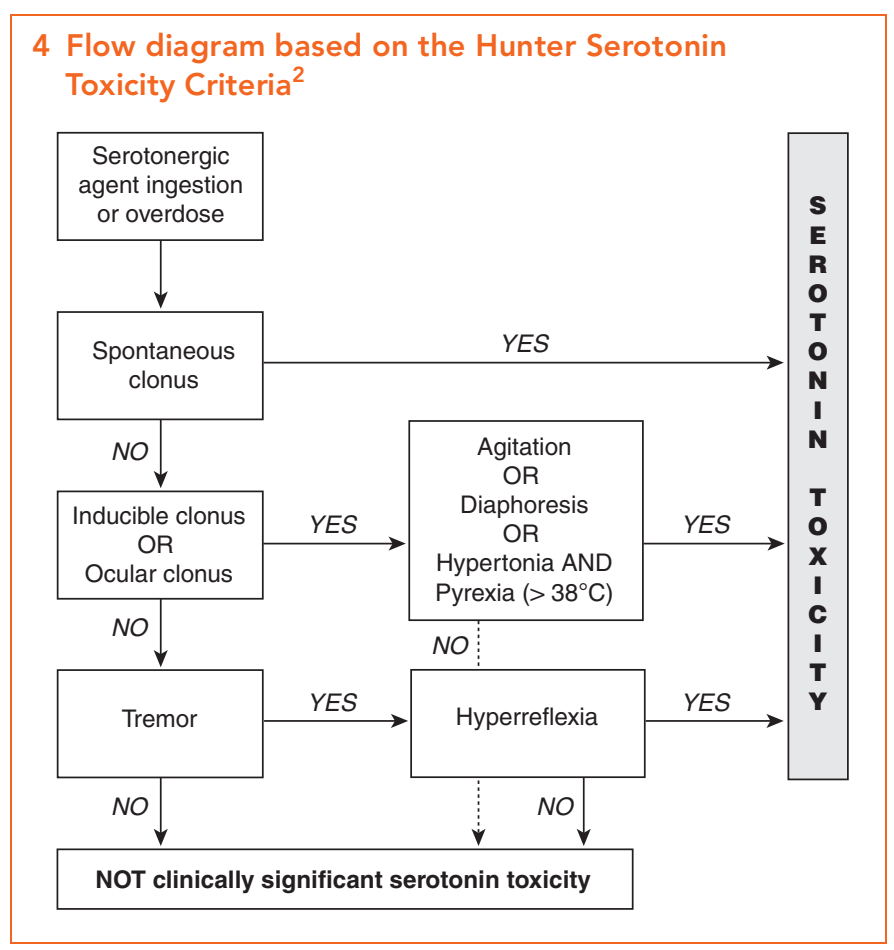

ity. Neuroleptic malignant syndrome is also an idiosyncratic adverse drug reaction that has a more gradual onset and is not associated with overdoses of neuroleptics.

Non-convulsive seizures (toxic ictal delirium) may present as a severe fluctuating acute confusional state, which may be associated with autonomic features, hyperreflexia or occasional overt seizure activity. Although the many non-drug causes of non-convulsive status epilepticus (including most causes of encephalopathy) ${ }^{10,11}$ must be excluded, drugs may induce this epileptic phenomenon, either dose-related or in withdrawal. The most common drug associations are with recently increased doses of phenothiazines and with benzodiazepine withdrawal. ${ }^{12}$ It seems likely that some serotonergic drugs may also occasionally cause this syndrome. An electroencephalogram at the time is usually diagnostic, and rapid improvement with administration of benzodiazepines is usual. The latter may be the best way to clinically distinguish non-convulsive seizures from serotonin toxicity. ${ }^{12}$

Acute baclofen withdrawal (typically due to intrathecal pump failure) is a potentially life-threatening condition that presents with a raised temperature, autonomic instability and muscle spasms and rigidity. ${ }^{13,14}$ The clinical features are difficult to distinguish from serotonin toxicity, but this rare diagnosis need only be considered in a few patients, and it should respond rapidly to reinstitution of baclofen. Interestingly, it has also been reported to respond to serotonin antagonists, ${ }^{14}$ suggesting that it may even be a specific form of serotonin toxicity.

\section{Severity of serotonin toxicity}

The spectrum of serotonin toxicity can be roughly divided into three groups of severity based on the requirement for medical intervention:

- Mild serotonergic features. These may or may not interfere with the patient and may occur with therapeutic use of many serotonergic agents.
- Moderate toxicity. Symptoms cause the patient significant distress and deserve symptomatic treatment.

- Severe serotonin toxicity or serotonin crisis. ${ }^{1}$ This is characterised by a rapidly increasing temperature associated with muscle rigidity, and will progress to multiorgan failure if not treated within hours. This is a medical emergency and is almost exclusively associated with combinations of drugs acting at different sites, most commonly including a monoamine oxidase inhibitor and an SSRI. ${ }^{15}$

A similar division was also suggested by Radomski and colleagues, labelled as mild serotonin toxicity, serotonin syndrome, and toxic states. ${ }^{16}$ A number of other scoring systems have been suggested; for example, a serotonin toxicity scale devised by Hegerl and coworkers for depressed patients taking paroxetine. ${ }^{17}$

Serotonin toxicity occurs in three main settings. Adverse reactions to normal therapeutic doses usually only cause mild-tomoderate toxicity. Overdose of a single serotonergic agent typically leads to moderate toxicity only. Nearly all severe serotonin toxicity relates to drug interactions (which may also occur in overdose).

There are a number of different mechanisms by which the drugs associated with serotonin toxicity (Box 1) cause excess serotonin. As mentioned above, severe serotonin toxicity mostly occurs with combinations of drugs (most commonly an SSRI and a monoamine oxidase inhibitor) acting at different sites. ${ }^{15}$ Patients who ingest any combination of serotonergic drugs in overdose must be observed carefully. However, not all combinations cause increased toxicity. For example, although methylenedioxymethamphetamine (MDMA; ecstasy) can cause serotonin toxicity, SSRIs do not appear to increase serotonergic effects from such serotonergic amphetamines and may reduce neurotoxicity. ${ }^{18,19}$

That single agents are unlikely to cause severe serotonin toxicity has been confirmed in studies of overdose patients ingesting SSRIs alone. ${ }^{2,3}$ In one study of SSRI overdoses, serotonin toxicity occurred in $15 \%$ of cases, but there were no severe cases. ${ }^{3}$

\section{Treatment}

Treatment for all forms of serotonin toxicity is supportive care and cessation of any serotonergic medications. Severe serotonin toxicity or serotonin crisis is a medical emergency and initial manage-

\section{Differential diagnoses for serotonin toxicity}

\begin{tabular}{|c|c|}
\hline Differ & Distinguishing features \\
\hline $\begin{array}{l}\text { Neuroleptic malignant } \\
\text { syndrome }\end{array}$ & $\begin{array}{l}\text { Absence of neuromuscular excitation;* } \\
\text { and presence of bradykinesia, lead-pipe } \\
\text { rigidity, and extrapyramidal features }\end{array}$ \\
\hline Non-convulsive seizures & $\begin{array}{l}\text { Electroencephalogram features, and } \\
\text { response to benzodiazepines }\end{array}$ \\
\hline $\begin{array}{l}\text { Acute baclofen } \\
\text { withdrawal }\end{array}$ & $\begin{array}{l}\text { History of intrathecal baclofen pump, } \\
\text { and response to baclofen }\end{array}$ \\
\hline $\begin{array}{l}\text { CNS infection - } \\
\text { encephalitis, meningitis }\end{array}$ & Absence of neuromuscular excitation* \\
\hline Anticholinergic delirium & $\begin{array}{l}\text { Absence of neuromuscular excitation, }{ }^{*} \\
\text { bowel sounds absent, and dry skin }\end{array}$ \\
\hline $\begin{array}{l}\text { Sympathomimetic } \\
\text { toxicity }\end{array}$ & Absence of neuromuscular excitation* \\
\hline Malignant hyperthermia & $\begin{array}{l}\text { Anaesthetic exposure, and absence of } \\
\text { neuromuscular excitation* }\end{array}$ \\
\hline
\end{tabular}

* Hyperreflexia, clonus and myoclonus.

CNS $=$ central nervous system. 
ment must focus on airway, breathing and circulation. Supportive care, including passive and active cooling of the patient, sedation, intubation and muscle paralysis, must take precedence over any specific pharmacological treatment. Hyperthermia and muscle rigidity appear to be the most important effects, and this supportive care can prevent secondary complications, such as rhabdomyolysis, renal failure and disseminated intravascular coagulation.

Serotonin toxicity may progressively increase over a number of hours after ingestion of implicated drugs. Patients who have moderate serotonin toxicity should be observed for a period of 6 hours; however, if a slow-release formulation has been ingested, such as venlafaxine, observation should be continued for 12 hours. It is appropriate to provide symptomatic treatment for these patients, including benzodiazepine sedatives, antiemetics and specific pharmacological therapy. Most patients will improve within 24 hours of ceasing the serotonergic medication.

There may be a role for specific serotonin antagonists in serotonin toxicity, and animal studies provide data that nonspecific $\mathrm{HT}_{2}$-antagonists and more selective $5-\mathrm{HT}_{2 \mathrm{~A}}$-antagonists reverse the lethal effects of serotonin toxicity. ${ }^{5}$ There are numerous case reports of patients improving after being given serotonin antagonists. ${ }^{20-24}$ However, it is difficult to separate these "responses" from the natural resolution of toxicity. There are no controlled trials demonstrating their effectiveness and further study is required. Cyproheptadine and chlorpromazine are the $\mathrm{HT}_{2}$-antagonists that have been used most extensively, ${ }^{20,23,25}$ and have a long history of safe use for other medical conditions. Oral cyproheptadine (4-12 mg) is probably the most useful $5-\mathrm{HT}_{2}$ antagonist for moderate toxicity. Its main side effect is sedation, which is usually beneficial. However, as cyproheptadine can only be administered orally, it is unlikely to be effective in patients administered activated charcoal, and has limited use in severe toxicity. In severe serotonin toxicity, chlorpromazine may be more appropriate to use for sedation than other routine sedative agents. It can cause hypotension, so patients must receive sufficient volume loading. Other non-selective $5-\mathrm{HT}_{2}$ antagonists, such as the atypical antipsychotics, may be effective, ${ }^{21,22}$ but there is far less experience with their use.

In patients who ingest overdoses of serotonergic agents, there are a few additional considerations. The selective use of activated charcoal may be warranted, but only if it can be given within an hour. Early risk assessment should also consider the possibility of interacting drugs, and non-serotonergic toxic effects (eg, QT prolongation with citalopram). ${ }^{26}$

Some individuals appear to be more susceptible to mild-tomoderate serotonin toxicity, but it is unclear whether this usually has a pharmacokinetic (eg, decreased drug metabolism) or pharmacodynamic (eg, serotonin receptor polymorphism) explanation. ${ }^{27}$ A great deal of research would be needed to enable identification of such individuals before treatment. Until then, prevention of serotonin toxicity is as simple (or as difficult) as avoiding prescribing serotonergic drugs. However, we believe that avoiding monoamine oxidase inhibitors may be sufficient to prevent life-threatening toxicity. It also makes sense to us to generally minimise the use of serotonergic drugs used for nonpsychiatric conditions.

\section{Competing interests}

None identified.

\section{Author details}

Geoffrey K Isbister, MB BS, MD, FACEM, Senior Research Fellow, and Clinical Toxicologist ${ }^{1,2,3}$

Nicholas A Buckley, BMed, MD, FRACP, Clinical Pharmacologist, and Associate Professor ${ }^{4}$

Ian M Whyte, MB BS, FRACP, Senior Staff Specialist, and Conjoint Professor ${ }^{2,3}$

1 Tropical Toxinology Unit, Menzies School of Health Research, Charles Darwin University, Darwin, NT.

2 Department of Clinical Toxicology and Pharmacology, Calvary Mater Newcastle Hospital, and University of Newcastle, Newcastle, NSW.

3 Discipline of Clinical Pharmacology and Toxicology, University of Newcastle, Newcastle, NSW.

4 Department of Clinical Pharmacology and Toxicology, Canberra Hospital, and Australian National University, Canberra, ACT.

Correspondence: gsbite@ferntree.com

\section{References}

1 Isbister GK, Buckley NA. The pathophysiology of serotonin toxicity in animals and humans: implications for diagnosis and treatment. Clin Neuropharmacol 2005; 28: 205-214.

2 Dunkley EJ, Isbister GK, Sibbritt D, et al. The Hunter Serotonin Toxicity Criteria: simple and accurate diagnostic decision rules for serotonin toxicity. QJM 2003; 96: 635-642.

3 Isbister GK, Bowe SJ, Dawson A, Whyte IM. Relative toxicity of selective serotonin reuptake inhibitors (SSRIs) in overdose. J Toxicol Clin Toxicol 2004; 42: 277-285.

4 McManus P, Mant A, Mitchell PB, et al. Recent trends in the use of antidepressant drugs in Australia, 1990-1998. Med J Aust 2000; 173: 458461.

5 Nisijima K, Yoshino T, Yui K, Katoh S. Potent serotonin (5-HT)(2A) receptor antagonists completely prevent the development of hyperthermia in an animal model of the 5-HT syndrome. Brain Res 2001; 890: 23-31.

6 Sternbach H. The serotonin syndrome. Am J Psychiatry 1991; 148: 705713.

7 Haslett CD, Kumar S. Can olanzapine be implicated in causing serotonin syndrome? Psychiatry Clin Neurosci 2002; 56: 533-535.

8 Whyte IM. Serotonin toxicity/syndrome. In: Medical toxicology. 3rd ed. Philadelphia: Lippincott Williams \& Wilkins, 2004: 103-106.

9 Shelton RC. The nature of the discontinuation syndrome associated with antidepressant drugs. J Clin Psychiatry 2006; 67 Suppl 4: 3-7.

10 Primavera A, Giberti L, Scotto P, Cocito L. Nonconvulsive status epilepticus as a cause of confusion in later life: a report of 5 cases. Neuropsychobiology 1994; 30: 148-152.

11 Walker MC. Diagnosis and treatment of nonconvulsive status epilepticus. CNS Drugs 2001; 15: 931-939.

12 van Sweden B, Mellerio F. Toxic ictal delirium. Biol Psychiatry 1989; 25 : 449-458.

13 Mohammed I, Hussain A. Intrathecal baclofen withdrawal syndrome - a life-threatening complication of baclofen pump: a case report. BMC Clin Pharmacol 2004; 4: 6

14 Meythaler JM, Roper JF, Brunner RC. Cyproheptadine for intrathecal baclofen withdrawal. Arch Phys Med Rehabil 2003; 84: 638-642.

15 Isbister GK, Hackett LP, Dawson AH, et al. Moclobemide poisoning: toxicokinetics and occurrence of serotonin toxicity. $\mathrm{Br} \mathrm{J}$ Clin Pharmacol 2003; 56: 441-450.

16 Radomski JW, Dursun SM, Reveley MA, Kutcher SP. An exploratory approach to the serotonin syndrome: an update of clinical phenomenology and revised diagnostic criteria. Med Hypotheses 1999; 55: 218-224.

17 Hegerl U, Bottlender R, Gallinat J, et al. The serotonin syndrome scale: first results on validity. Eur Arch Psychiatry Clin Neurosci 1998; 248: 96103.

18 Malberg JE, Sabol KE, Seiden LS. Co-administration of MDMA with drugs that protect against MDMA neurotoxicity produces different effects on body temperature in the rat. J Pharmacol Exp Ther 1996; 278: 258-267.

19 Sanchez V, Camarero J, Esteban B, et al. The mechanisms involved in the long-lasting neuroprotective effect of fluoxetine against MDMA 
("ecstasy")-induced degeneration of 5-HT nerve endings in rat brain. Br J Pharmacol 2001; 134 : 46-57.

20 Graudins A, Stearman A, Chan B. Treatment of the serotonin syndrome with cyproheptadine. J Emerg Med 1998; 16: 615-619.

21 Boddy R, Ali R, Dowsett R. Use of sublingual olanzapine in serotonin syndrome [abstract]. J Toxicol Clin Toxicol 2004; 42: 725.

22 Boddy R, Dowsett RP, Jeganathan D. Sublingual olanzapine for the treatment of serotonin syndrome [abstract]. Clin Toxicol 2006; 44: 426.

23 Chan BS, Graudins A, Whyte IM, et al. Serotonin syndrome resulting from drug interactions. Med J Aust 1998; 169: 523-525.

24 Graham PM. Successful treatment of the toxic serotonin syndrome with chlorpromazine [letter]. Med J Aust 1997; 166: 166-167.

25 Gillman PK. The serotonin syndrome and its treatment. J Psychopharmacol 1999; 13: 100109.

26 Isbister GK, Friberg LE, Duffull SB. Application of pharmacokinetic-pharmacodynamic modelling in management of QT abnormalities after citalopram overdose. Intensive Care Med 2006; 32: 1060-1065.

27 Murphy GM, Kremer C, Rodrigues HE, Schatzberg AF. Pharmacogenetics of antidepressant medication intolerance. Am J Psychiatry 2003; 160: 1830-1835.

(Received 29 Mar 2007, accepted 25 Jun 2007)

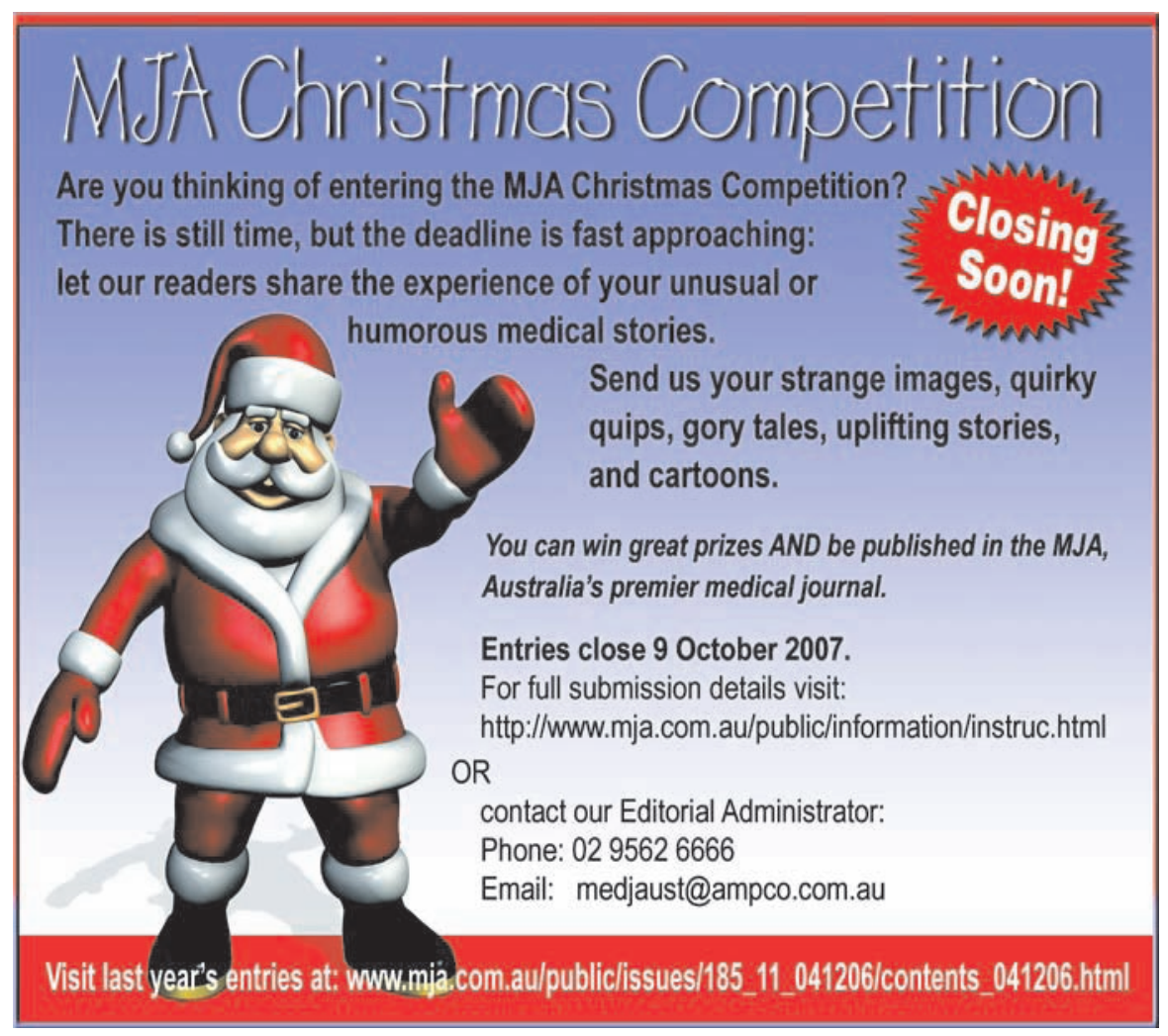

